\title{
Microwave-Assisted Solvent-Free Synthesis of Zeolitic Imidazolate Framework-67
}

\author{
Heng Zhang, ${ }^{1,2,3}$ Jing Zhong, ${ }^{1,2,3}$ Guoxiang Zhou, ${ }^{3}$ Junliang $W u,{ }^{3}$ \\ Zhenyu Yang, ${ }^{4}$ and Xianming $\mathrm{Shi}^{5}$ \\ ${ }^{1}$ Key Laboratory of Structure Dynamic Behavior and Control (Harbin Institute of Technology), Ministry of Education, Harbin, \\ Heilongjiang 150090, China \\ ${ }^{2}$ School of Civil Engineering, Harbin Institute of Technology, Harbin 150090, China \\ ${ }^{3}$ School of Civil Engineering and Architecture, Wuhan Polytechnic University, Wuhan 430023, China \\ ${ }^{4}$ Department of Chemistry, Nanchang University, Nanchang 330031, China \\ ${ }^{5}$ Department of Civil \& Environmental Engineering, Washington State University, P.O. Box 642910, Pullman, WA 99164-2910, USA
}

Correspondence should be addressed to Jing Zhong; zhongjing@hit.edu.cn and Xianming Shi; xianming.shi@wsu.edu

Received 17 December 2015; Revised 3 March 2016; Accepted 13 March 2016

Academic Editor: Gurvinder Singh

Copyright (C) 2016 Heng Zhang et al. This is an open access article distributed under the Creative Commons Attribution License, which permits unrestricted use, distribution, and reproduction in any medium, provided the original work is properly cited.

\begin{abstract}
A microporous metal-organic framework (MOF), cobalt-based zeolitic imidazolate framework-67 (ZIF-67), was synthesized by the combination of solvent-free hand-mill and microwave irradiation, without any organic solvent and within 30 minutes. The hand-milling process can mix the reactants well by the virtue of high moisture/water absorption capacity of reactants. In addition, the outstanding electromagnetic wave absorption capability of cobalt leads to efficient conversion to MOF structures before carbonization. The obtained ZIF-67 possesses high surface area and micropore volume.
\end{abstract}

\section{Introduction}

Metal-organic frameworks (MOFs) are a family of highly porous crystalline materials, which consist of metal ions and ligands that are coordinated into 3D structures. In light of their unique physicochemical characteristics, MOFs have great potential for applications such as gas storage $[1,2]$, separation [3], drug delivery [4], heterogeneous catalysts [5], luminescence [6], magnetism [7], biomedical sensors [8], and heating transformation through reversible water adsorption and desorption [9]. MOFs feature very large specific surface area and pores size controllable and adjustable by the proper selection of metal ions and organic linkers $[10,11]$. In addition, the organic linkers can be functionalized independently without sacrificing the geometrical characteristics of $3 \mathrm{D}$ porous structures, which is another unique advantage $[12,13]$.

Although MOFs have garnered increasing interests of researchers, there are still several factors limiting their practical applications $[14,15]$. The use of organic solvents and long synthesis time are two of the top challenges. Organic solvents pose the risk to operators and the natural environment, leading to limited autoclave availability, whereas the long synthesis time tends to translate to high energy consumption and cost.

There are several strategies to address challenges in synthesizing MOFs, among which the solvent-free (SF) synthesis and microwave (MW) synthesis are very promising [16, 17]. For instance, Lin et al. reported that the porous metal azolate framework can be synthesized by simply heating the mixture of metal oxide/hydroxide and azole ligand only [18]. Friscic adopted "accelerated aging" to assist the synthesis of multigram of metal-organic framework without using solvents, high temperature, or another activation [19]. Nonetheless, with few exceptions [20], SF mixing alone without any subsequent or concurrent treatment, such as hydrothermal process, would not effectively produce MOFs. While it is essential to mix the solid reactants well in order to synthesize uniform MOF particles, this can only be partially achieved 
with the aid of ball milling [21, 22]. Microwave-assisted synthesis of MOFs has proven its effectiveness in recent years $[23,24]$. Microwave absorption can induce vibration of atoms and molecules and thus convert mechanical energy to thermal energy, which induces chemical reactions very efficiently [25]. The polar solvents, which have high dielectric absorptivity, function as thermal conversion agents to eliminate local superheat spots, promoting fast and homogeneous nucleation over the MOF growth process. In this study, a hypothesis to test is that the efficiency of MW treatment should also depend on the wave absorption capability of the metal ions. This factor, to the best of our knowledge, has not been considered in previous studies.

Zeolitic imidazolate frameworks (ZIFs) are a class of MOFs that are topologically isomorphic with zeolites, in which tetrahedrally coordinated transition metal ions are connected by imidazole linkers [26]. The structure of ZIFs is highly stable, and it can be heated to high temperatures without decomposing, making them suitable for use in hot environments [27]. Tanaka et al. reported a mechanochemical dry conversion of $\mathrm{ZnO}$ to ZIF-8 by ball milling, which avoids the use of organic solvents using excess amount of 2-methylimidazole. And the BET surface area of obtained ZIF-8 reached the maximum $\left(1480 \mathrm{~m}^{2} \mathrm{~g}^{-1}\right)$ after milling for $96 \mathrm{~h}$ [28]. Subsequently, Lanchas et al. used 1-butanol, pyridine, and 4-methylpyridine as structure directing agents to generate ZIF single crystals above $88-90^{\circ} \mathrm{C}$ [29]. Lin et al. also reported that the porous metal azolate framework can be synthesized by heating the mixture of metal oxide/hydroxide and azole ligand at $100^{\circ} \mathrm{C}$ for $24 \mathrm{~h} \mathrm{[18]}$. Although it has been reported that ZIF-67 can be obtained within several minutes by hydrothermal methods, either excess 2-methylimidazole $\left(\mathrm{Zn}^{2+}: 2\right.$-methylimidazole $\left.: \mathrm{H}_{2} \mathrm{O}=1: 70: 1238\right)$ or additives $[30,31]$, such as trimethylamine, are needed, which results in the waste of reactants and pollution to the environment. In addition, the samples synthesized within minutes by normal solvothermal/hydrothermal methods generally have lower surface areas and smaller pore volumes. A mechanochemical synthesis of ZIFs in which crystal formation can be completed within several minutes has not been reported. Interestingly, we also noticed that the cobalt ions in ZIFs have excellent electromagnetic wave absorption properties [32-34] and thus may facilitate the fast synthesis of ZIFs via the MW treatment method.

Here, we describe a very rapid method for the synthesis of cobalt-based zeolitic imidazolate framework-67 (ZIF-67), taking the advantages of coupling SF and MW treatments. The goal is to eliminate the use of organic solvent in the process and shorten the synthesis time to less than 30 minutes, including 20 minutes for manual grinding and 5 minutes for the crystallization.

\section{Experimental Section}

2.1. Materials. All the reagents, including cobalt nitrate hexahydrate (98\%, Sigma-Aldrich), 2-methylimidazole (99\%, Sinopharm), and cobalt nitrate hexahydrate (98\%, SigmaAldrich) were used as received.
2.2. Preparation of ZIF-67. The preparation of ZIF-67 consisted of three steps: (1) mixing the starting materials by mechanical attrition at room temperature for $20 \mathrm{~min}$; (2) production of ZIF-67 crystals with MW-irradiation; (3) washing the as-synthesized ZIF-67 with methanol for 3 times. Specifically, $895 \mathrm{mg}$ 2-methylimidazole was finely powdered in an agate mortar for $5 \mathrm{~min}$; then $404.8 \mathrm{mg} \mathrm{Co}\left(\mathrm{NO}_{3}\right)_{2} \cdot 6 \mathrm{H}_{2} \mathrm{O}$ was added and the mixture was ground for another $15 \mathrm{~min}$. The resulting mixture was transferred into a quartz boat and placed in a domestic microwave oven and heated for $5 \mathrm{~min}$. The obtained solid, termed SF_MW ZIF-67, was washed with methanol for 3 times and then separated by centrifugation. Domestic microwave oven (G90W25M SP-WD) was used, with the power of $900 \mathrm{~W}$.

We also tested whether the addition of cobalt-containing salt to the precursors of Zn-based MOFs can facilitate the formation of Zn-based MOFs. We prepared ZIF-8 with the solvent-free method by adding small amount of cobalt source $\left(\mathrm{Co}\left(\mathrm{NO}_{3}\right)_{2} \cdot 6 \mathrm{H}_{2} \mathrm{O}\right)$ into the starting materials. $785 \mathrm{mg}$ of 2 methylimidazole was ground for 5 minutes, followed by the addition of $642 \mathrm{mg}$ of $\mathrm{Zn}\left(\mathrm{NO}_{3}\right)_{2} \cdot 6 \mathrm{H}_{2} \mathrm{O}$. Then $166 \mathrm{mg}$ of $\mathrm{Co}\left(\mathrm{NO}_{3}\right)_{2} \cdot 6 \mathrm{H}_{2} \mathrm{O}$ was added and ground for another 15 minutes. The microwave treatment is the same as that of the SF_MW ZIF-67.

2.3. Characterization. The structures of the ZIF-67 crystals were investigated by scanning electron microscopy (HELIOS Nanolab 600i); X-ray diffraction (XRD, Empyrean series 2, PANalytical) and infrared spectrum (IR, Frontier Optical, PE) surface areas were measured by $3 \mathrm{H}-2000 \mathrm{PS} 1$ Specific \& Pore Size Analysis Instrument (BeiShiDe Instrument).

\section{Results and Discussion}

During the solvent-free (SF) synthesis of MOF, mechanical grinding is required to promote the reactions between solid reactants through multiple mechanisms, including heating, defects formation, particle size reduction, local melting, and even phase changes to other polymorphs [32]. Ball mill is generally preferred to grind the solid reactants, since it can supply high power, being programmable, and result in smaller particles with small polydispersity [17]. In this study, however, instead of ball mill, we use hand-mill only to accelerate the moisture/water absorption of the solid reactants. Once the two reactants are mixed and ground, the solid mixture turns into blue liquid (Figures 1(a) and 1(b)) within several minutes (which is dependent on the air humidity). It is mainly caused by the high moisture absorption capability of cobalt nitrate hexahydrate. The quantity of absorbed moisture seems large enough to dissolve all reactants (cobalt nitrate hexahydrate and 2-methylimidazole) without any noticeable solid particles, making the reactants well mixed. Upon further hand-milling for several minutes, solid pink particles (Figure 1(c)) gradually precipitated until the whole liquid mixture eventually changed back into solid particles. This is because 2-methylimidazole can gradually absorb water in the liquid mixture as long as it is not saturated. Such solid-liquid-solid phase change during hand-milling 


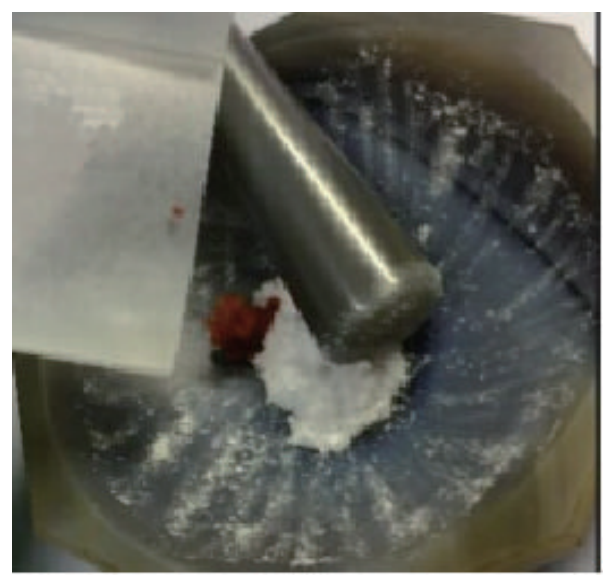

(a)

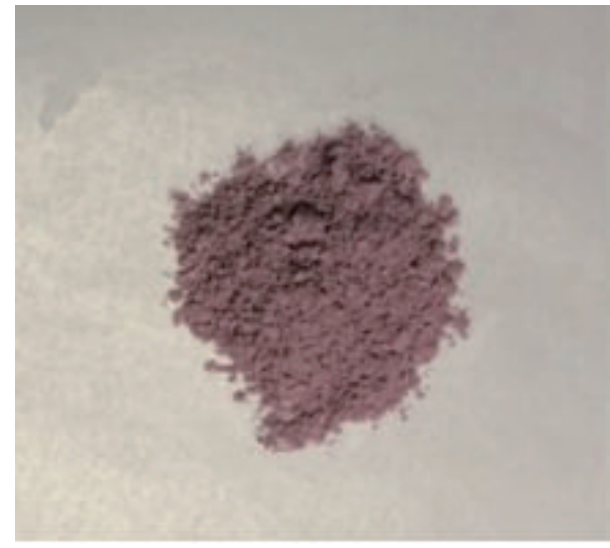

(c)

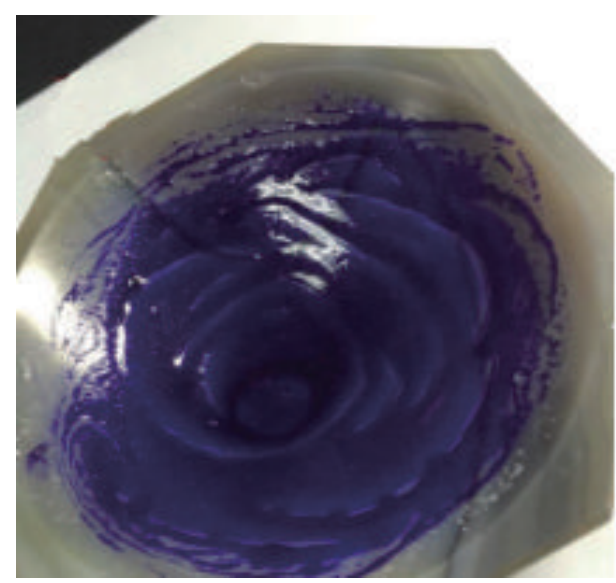

(b)

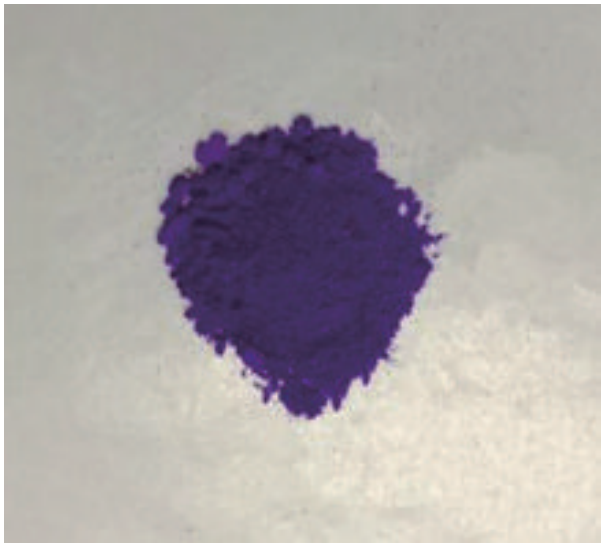

(d)

FIGURE 1: Photographs of starting materials (a), the liquid mixtures (b), and products before MW-irradiation (c) and after MW-irradiation (d).

can greatly facilitate the unprecedented uniform mixing of the solid reactants. Since the input energy to the reactants during hand-milling is negligible comparing to ball milling process, the mechanism related to the mechanochemical synthesis probably cannot be applied to the situation of handmilling. Indeed, as will be discussed in the following, the obtained solid particles after hand-milling do not possess any structural characteristics of ZIF-67. Those pink particles are then treated by MW-irradiation, which is necessary for the synthesis of ZIF-67 in this study. Upon MW treatment, it can be observed that the color of the particles changed from pink to purple (Figures $1(\mathrm{c})$ and $1(\mathrm{~d})$ ). It is worth pointing out that although the MW oven we used in this study is domestic and cheap instead of programmable and expensive industry MW oven, the physical/chemical properties of the obtained ZIF-67 are quite stable for more than five times synthesis and confirm the reproducibility.

Figure 2 presents SEM images of the ZIF-67 samples synthesized by the SF method. The samples maintain the rhombic dodecahedral shape, which is consistent with ZIF67 prepared by autoclaved method. However, the average size of the SF samples is about $500 \mathrm{~nm}$, which is much

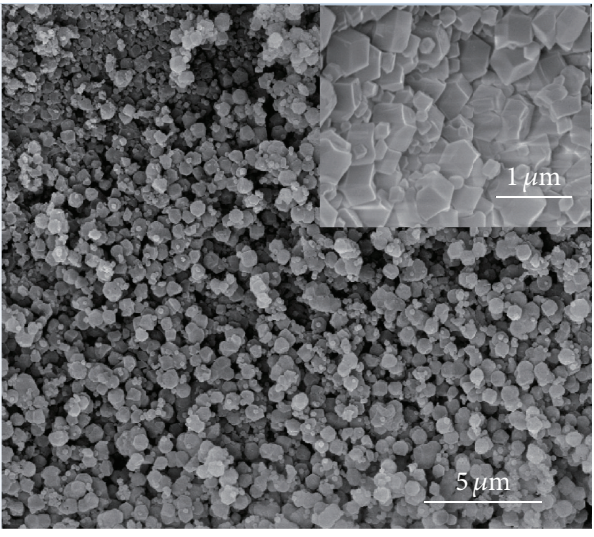

FIGURE 2: SEM images of ZIF-67 crystals.

smaller than the traditional one. This may be due to its short synthesis time resulting in a fast nucleation and short growth rate during the SF process. Figure 3 shows the structures of ZIF-67 at different MW-irradiation time. The structure 


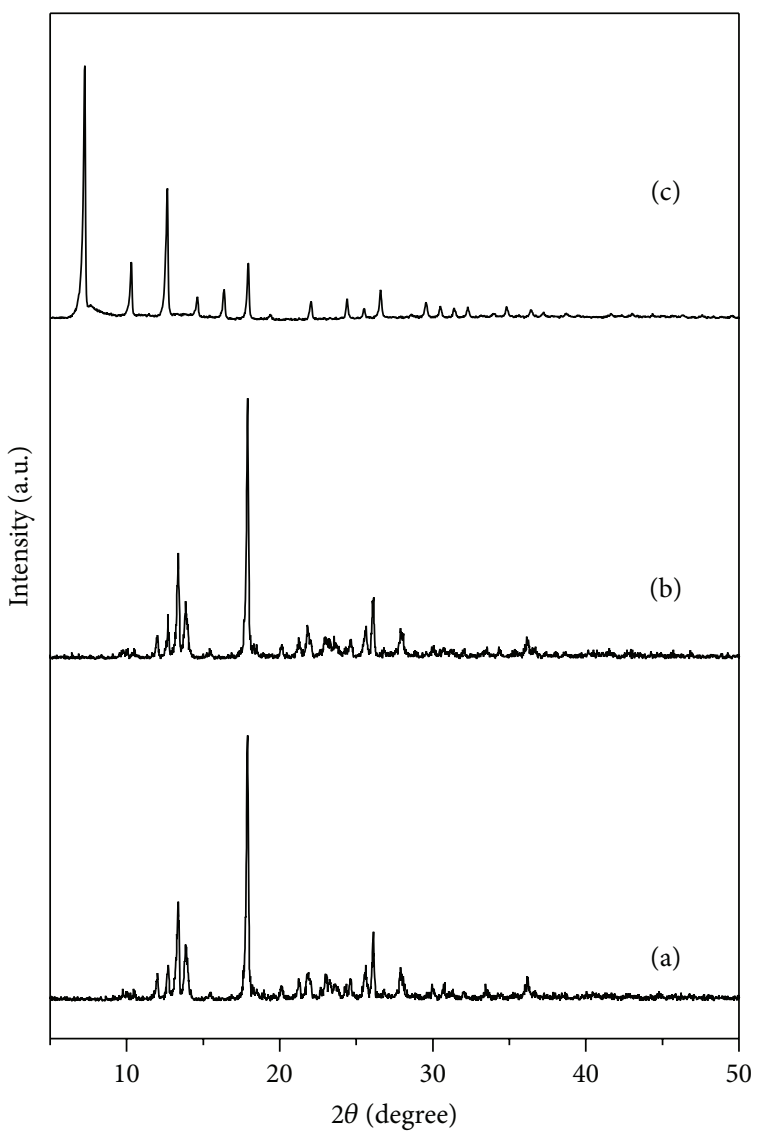

FIGURE 3: XRD patterns of as-synthesized ZIF-67 products under MW-irradiation for $0 \mathrm{~min}(\mathrm{a}), 1 \mathrm{~min}(\mathrm{~b})$, and $5 \mathrm{~min}$ (c).

and high crystallinity of our prepared ZIF-67 product are confirmed by the XRD patterns shown in Figure 3(c). The positions of diffraction peaks agree well with the simulated one. A favorable orientation of (110) direction was observed, from the highest peak at 2 theta value of 7.3 degrees, which represents (110) plane of ZIF-67 crystals. We can see from Figures 3(a) and 3(b) that, with no or not enough MW-irradiation, the peaks of the products do not conform to the simulated one, indicating no ZIF-67 structure was generated. This phenomenon was further certified by FTIR spectroscopy (Figure 4). From the comparison between the samples before and after MW-irradiation, we can see that, before microwave-assisted heating, the as-synthesized ZIF67 precursors obtained by just grinding exhibit peaks in accordance with 2-methylimidazole as reported in literature [35], implying no ZIF-67 product was prepared. However, after MW treatment, a new absorption band at $477 \mathrm{~cm}^{-1}$ was displayed, manifesting the Co-N stretching, which proved the existence of ZIF-67 structure. On the other hand, the duration of MW-irradiation should be carefully controlled to prevent the overheating of the particles. In this study, the samples are cooled down after each 1 min in MW treatment for the prevention of the collapse of the framework and even its carbonization.

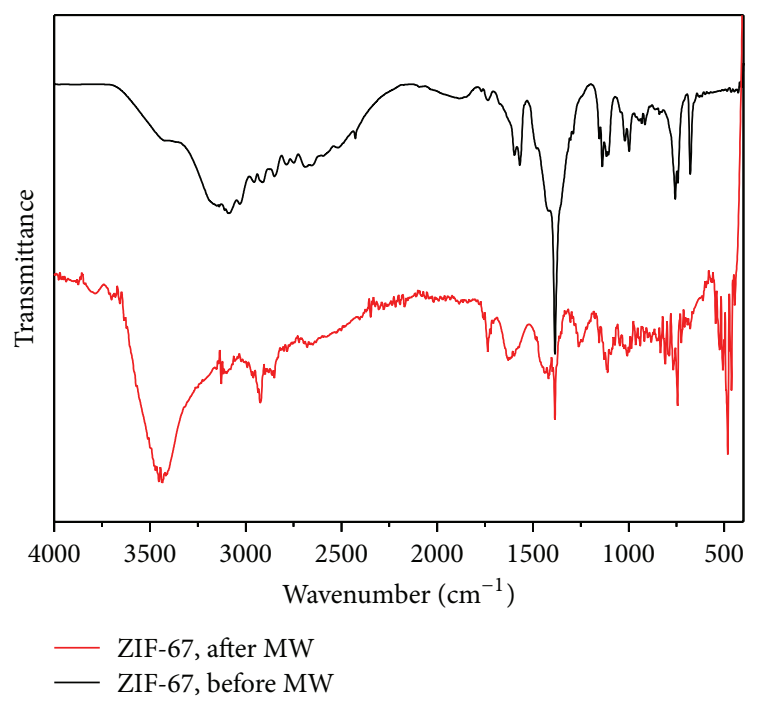

FIgURE 4: IR spectrum of SF_MW ZIF-67 crystals.

Figure 5(a) shows nitrogen adsorption isotherm of SF_MW ZIF-67, which is a Type I isotherm, representing a micropore structure. The Brunauer-Emmett-Teller (BET) specific surface area and Langmuir specific surface area of ZIF-67 are calculated to be $1120 \mathrm{~m}^{2} / \mathrm{g}$ and $1562 \mathrm{~m}^{2} / \mathrm{g}$, respectively. A pore size distribution ranging from $1.24 \mathrm{~nm}$ to $1.36 \mathrm{~nm}$, based on the Horvath-Kawazoe $(\mathrm{H}-\mathrm{K})$ model, is shown as in Figure 5(b). The peak at $1.3 \mathrm{~nm}$ probably resulted from the distortion of the second-building unit (SBU), which is formed through the reaction of $\mathrm{Co}^{2+}$ and 2-MIm during the hand mixing or microwave radiation. Considerable energy will be absorbed by the reaction system during the microwave radiation treatment, and it not only accelerates the formation of MOF but also might be able to distort the already formed SBU. As can be seen from the complementary experiments, in which the microwave radiation time decreased from 1 minute to 30 seconds, the size of the pores decreases obviously and the peak at $1.3 \mathrm{~nm}$ disappears. Clearly, the microwave radiation treatment time has some impact on the pore size distribution.

For the solvent-free microwave heating method in this study, we prepared ZIF-67 with the reactant quantity much closer to stoichiometric Co-2MI ratio (1:7.94), in which not only does it improve the reagent utilization efficiency and avoid using of poison reagents, but also the resultant MOFs have higher surface area $\left(1120 \mathrm{~m}^{2} / \mathrm{g}\right)$ and micropore volume $(0.52 \mathrm{~mL} / \mathrm{g})$. Actually, those values are similar to the MOFs obtained by traditional solvothermal/hydrothermal samples ( $>100^{\circ} \mathrm{C}, 1 \mathrm{~h}$ to $\left.1 \mathrm{month}\right)$. We further carried out experiments by shortening the heating time during each heating and cooling process. It was found that when the heating time decreases from 1 minute to 30 seconds (Figure 6), the surface area and micropore volume can be enhanced to $1591 \mathrm{~m}^{2} / \mathrm{g}$ and $0.74 \mathrm{~cm}^{3} / \mathrm{g}$, respectively, which fully testified the accessibility of this approach and, by optimizing the preparation conditions, we can synthesize ZIF-67 solids with good quality within several minutes but without using any 


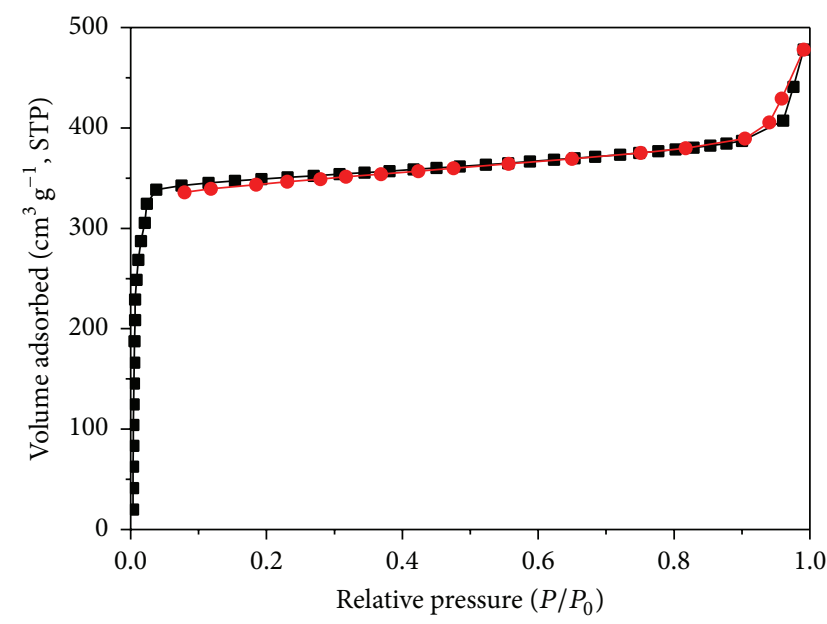

(a)

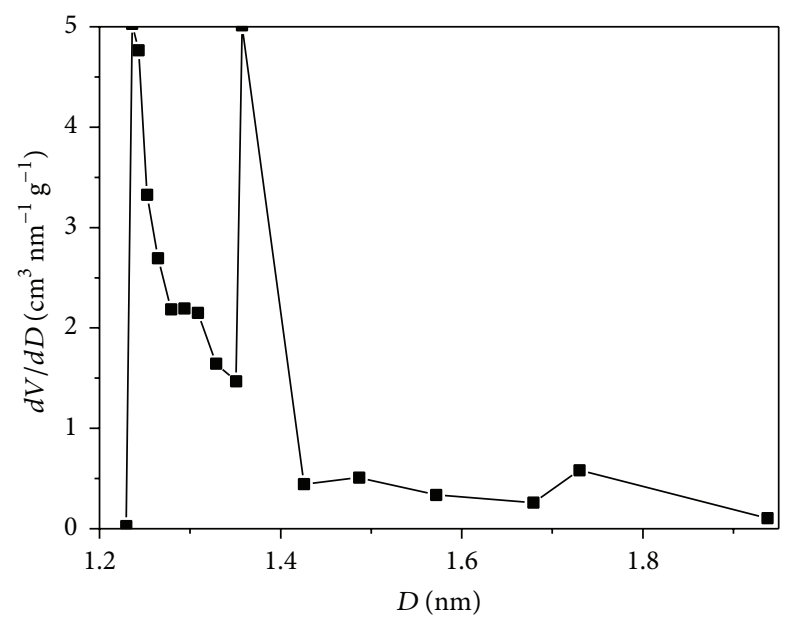

(b)

FIGURE 5: $\mathrm{N}_{2}$ adsorption-desorption isotherms (a) and H-K micropore size distribution (b) of SF_MW ZIF-67.

TABLE 1: Physical properties of different samples.

\begin{tabular}{lccc}
\hline Samples & Mean pore size $(\mathrm{nm})$ & $S_{\text {BET }}\left(\mathrm{m}^{2} / \mathrm{g}\right)$ & ${\text { Micropore volume }\left(\mathrm{cm}^{3} / \mathrm{g}\right)}$ \\
\hline C-ZIF-67 & 1.17 & 1725 & 0.82 \\
SF_MW ZIF-67-1 & 1.18 & 1120 & 0.52 \\
SF_MW ZIF-67-2 & 1.31 & 1591 & 0.74 \\
\hline
\end{tabular}

C-ZIF-67, ZIF-67 samples synthesized by the conventional solvothermal method.

SF_MW ZIF-67-1, ZIF-67 samples synthesized by the SF_MW method, in which heating time is 1 minute.

SF_ZIF-67-2, ZIF-67 samples synthesized by the SF_MW method, in which heating time is $30 \mathrm{~s}$.

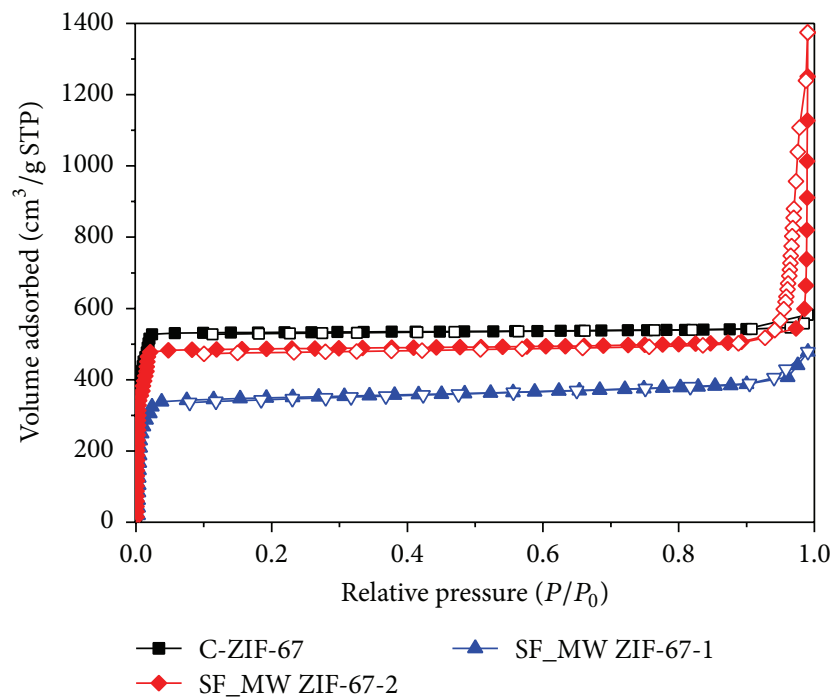

FIGURE 6: $\mathrm{N}_{2}$ adsorption isotherms of different samples.

poisonous reagent or waste of reactants. We also compare the porous structures of ZIF-67 obtained via MW treatment with conventional solvothermal method. From Figure 7, the lattice distance of the SF_MW ZIF-67 increased, while the particle size reduced from $800 \mathrm{~nm}$ to $500 \mathrm{~nm}$, implying that the pore size increased through MW treatment. This was confirmed by the pore size distribution by $\mathrm{N}_{2}$ adsorption testing in Table 1 , which shows a pore size distribution ranging from $1.24 \mathrm{~nm}$ to $1.36 \mathrm{~nm}$ and a median pore diameter of $1.31 \mathrm{~nm}$. This is much larger than the conventional ones with a median pore diameter of $1.17 \mathrm{~nm}$ and pore volume (at $P / P_{0}=0.024$ ) of $0.82 \mathrm{~cm}^{3} / \mathrm{g}$. In addition, the pore volume of the MW samples can be increased by tuning the heating time from 1 minute to $30 \mathrm{~s}$ (total $\mathrm{MW}$ heating time is the same, i.e., $5 \mathrm{~min}$ ) in every MW heating interval $\left(63 \%, P / P_{0}=0.038,0.52 \mathrm{~cm}^{3} / \mathrm{g}\right.$ versus $\left.90 \%, P / P_{0}=0.021,0.74 \mathrm{~cm}^{3} / \mathrm{g}\right)$. Meanwhile, the mean pore size also decreases to $1.18 \mathrm{~nm}$, which is similar with the traditional ones. This phenomenon illustrates that the porosity can be tuned by varying the heating intervals during the SF_MW process, which can facilitate the generation of hierarchical pores.

We also apply the same procedure to synthesize ZIF-8. However, MW-irradiation directly converts the particles into amorphous carbon instead of ZIF-8, and the addition of extra water to the precursors of ZIF-8 during hand mix still cannot prevent the carbonization (Figure 8). Further, we testified whether our method applies to those ZIF or MOF systems that can only be synthesized in solvothermal conditions, in which we tried the synthesis of MOF-5. Unfortunately, there is no MOF-5 generated according to the XRD analysis (Figure 9). Such distinct comparison clearly illustrates the importance of electromagnetic wave absorption capability of metal ions. Cobalt is one of the most effective electromagnetic wave absorbents. When irradiated by electromagnetic waves, cobalt ions act as superheat spots and trigger 


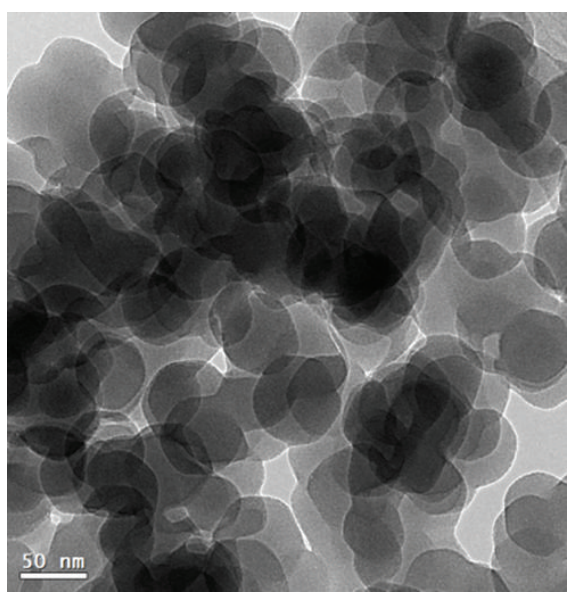

(a)

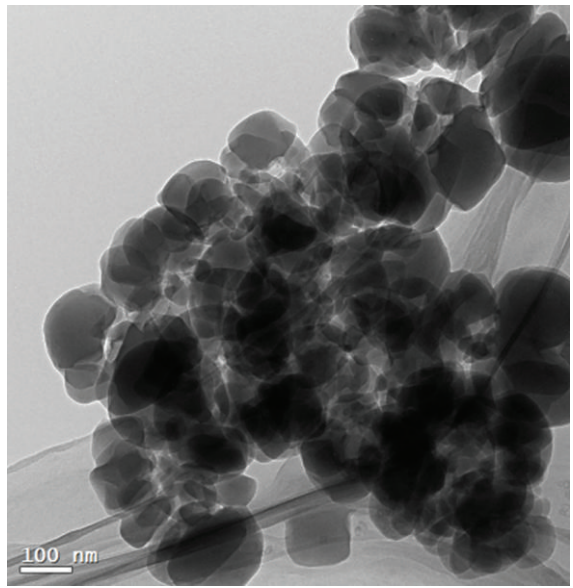

(d)

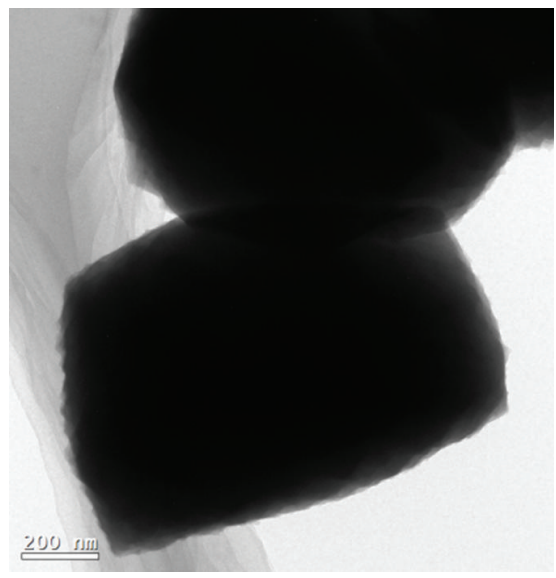

(g)

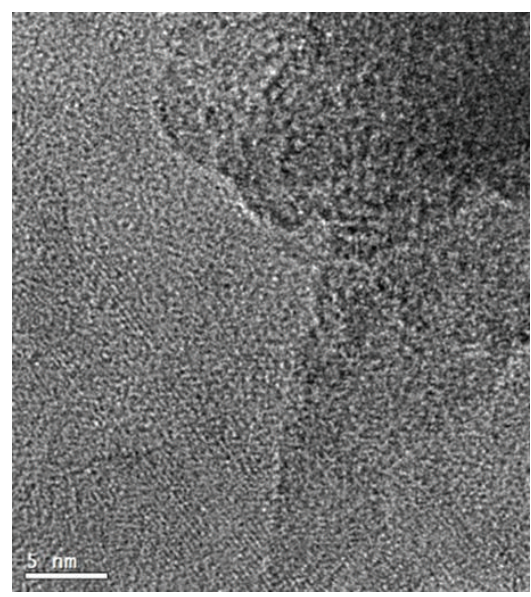

(b)

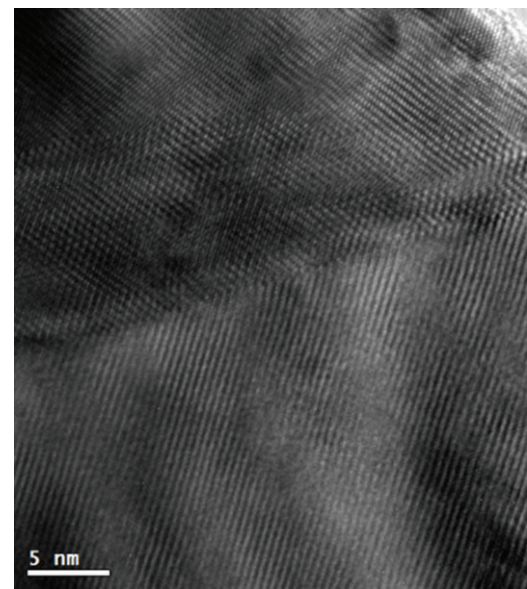

(e)

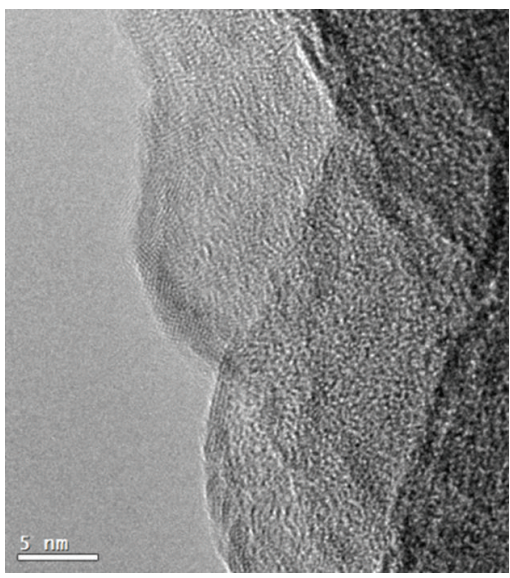

(h)

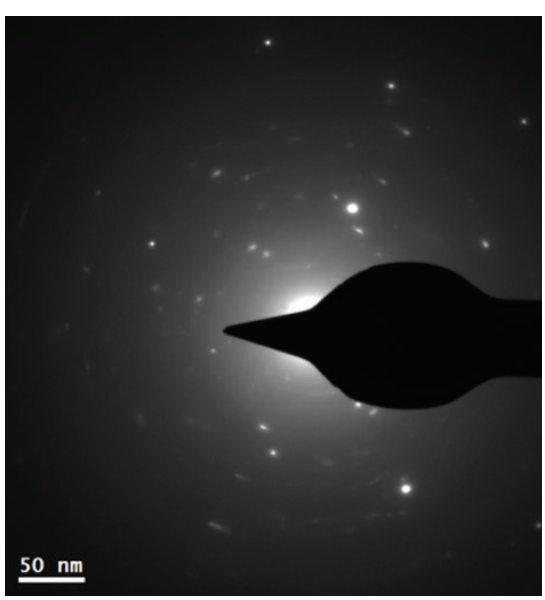

(c)

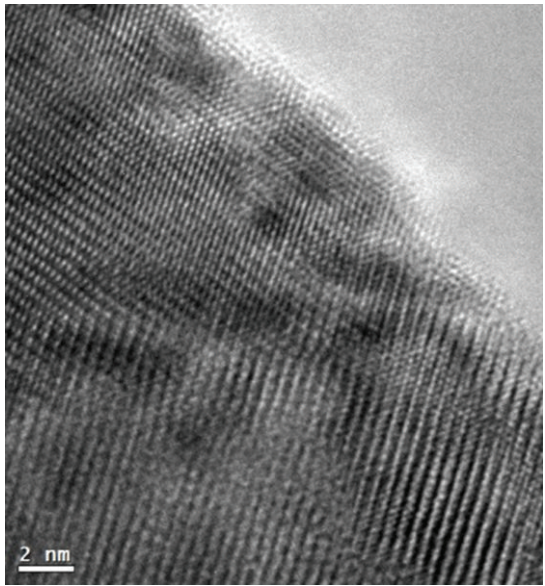

(f)

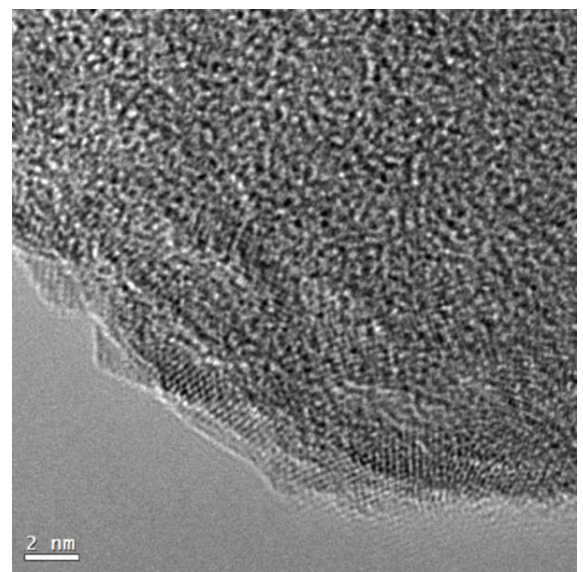

(i)

FIGURE 7: TEM images of the as-synthesized SF_MW ZIF-8 particles (a, b, c), SF_MW ZIF-67 particles (d, e, f), and C-ZIF-67 particles (g, h, i).

the complexation between the metal ions and ligands to form stable 3D microporous MOF structures. Therefore, the MOF formation mechanism during MW treatment in this study should be very different from the conventional ones where it is the organic solvent that acts as heating spot as we discussed previously. In addition, we expected that the existence of cobalt would facilitate the production of ZIF-8 due to its microwave-absorbing properties. We added a minimum of $\mathrm{Co}\left(\mathrm{NO}_{3}\right)_{2} \cdot 6 \mathrm{H}_{2} \mathrm{O}$ to the precursors for the synthesis of ZIF-8, and the results of this experiment testified 


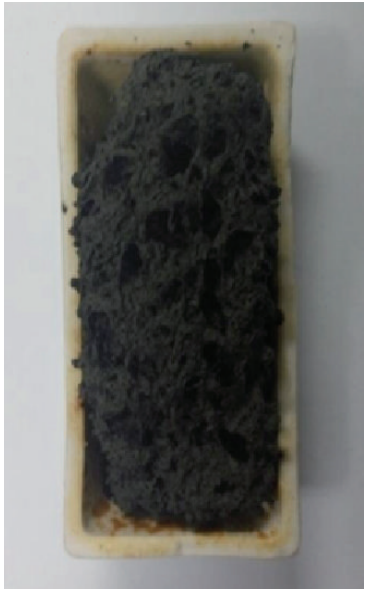

(a)

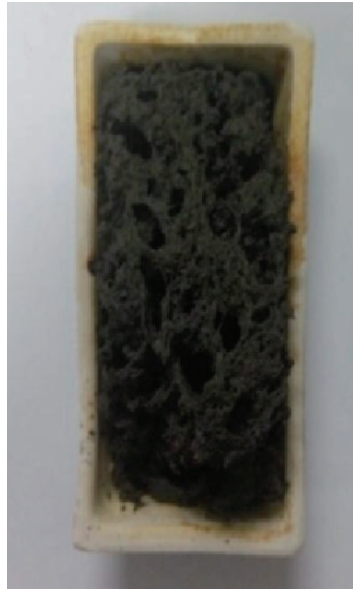

(b)

FIGURE 8: Images for the samples obtained by the microwave radiation for the precursors of ZIF-8 with extra (a) $10 \mathrm{~mL}$ water and (b) $100 \mathrm{~mL}$ water.

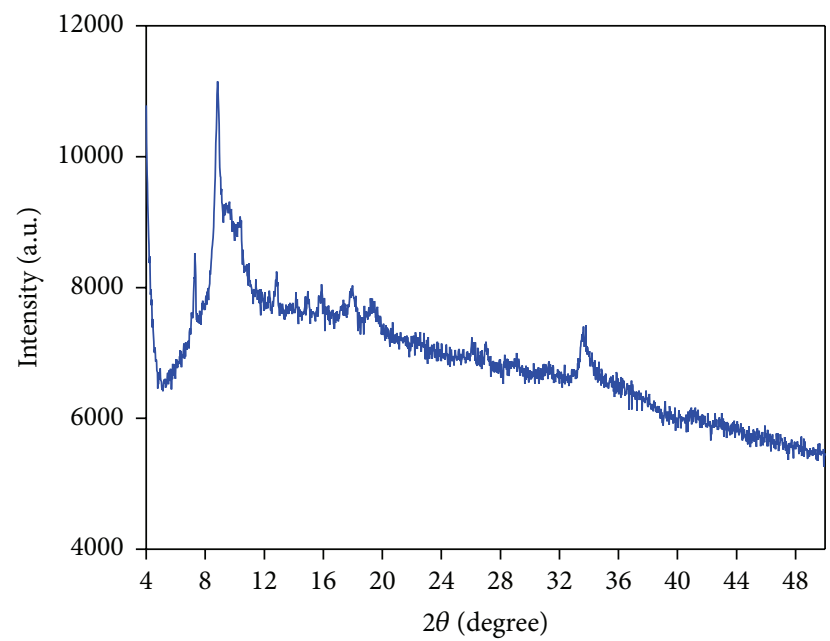

FIGURE 9: XRD for the samples obtained by hand mixing and microwave treatment for the precursors of MOF-5.

our assumption. It can be clearly seen from Figure 10 that there is a peak at $417 \mathrm{~cm}^{-1}$ in the IR spectrum of SF_MW ZIF8/ZIF-67, which represented the Zn-N stretching, implying the formation of ZIF-8 structure. When compared to the IR spectrum of SF_MW ZIF-67, the difference was evident between $400 \mathrm{~cm}^{-1}$ and $500 \mathrm{~cm}^{-1}$. In SF_MW ZIF-67, as exemplified in Figure 4, the adsorption band is at $477 \mathrm{~cm}^{-1}$, which is the characteristic for Co-N stretching. Therefore, ZIF-8 was prepared successfully with the existence of cobalt nitrate. The TEM images in Figure 7 (a) revealed that the particle size of ZIF-8 was about $50 \mathrm{~nm}$ with hexagonal shape.

\section{Conclusions}

In this study, we combine hand-milling with MW-irradiation methods to synthesize ZIF-67. This avoids the use of organic

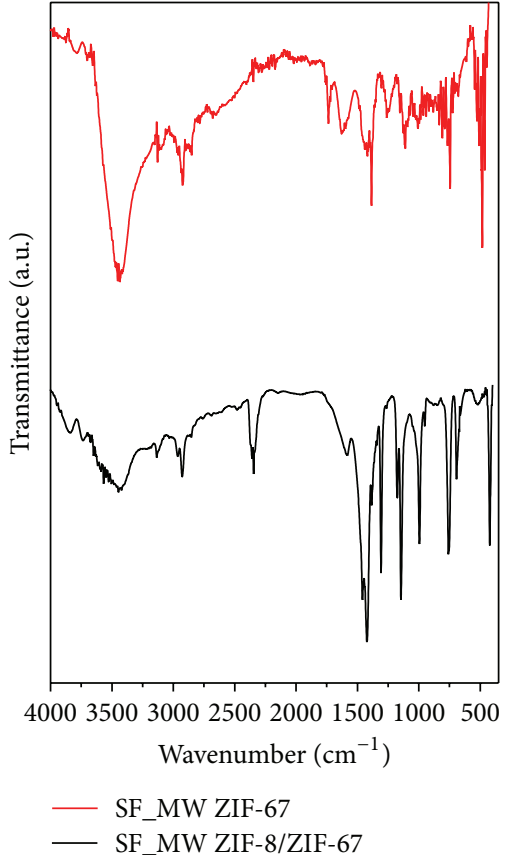

FIGURE 10: IR spectrum of SF_MW ZIF-67 (red) and SF_MW ZIF8/ZIF-67 (black).

solvent and shortens the synthesis time to less than $30 \mathrm{~min}$ utes. The hand-milling process is to mix the solid reactants well, whereas the MW-irradiation successfully converts the participating reactant particles to ZIF-67. The synthesized ZIF-67 features high surface area and micropore volume. We also show that the electromagnetic wave absorption capability of metal ions plays a key role in the successful conversion of reactants to MOFs, which merits further investigation. 


\section{Competing Interests}

The authors have confirmed that there are no competing interests related to this paper.

\section{Acknowledgments}

The research was financially supported by the National Science Foundation of China (no. 51402074 and no. 21263016), Jiangsu Postdoctoral Science Foundation (1402129C), the Six Talent Peaks Program of Jiangsu Province (2013-ZBZZ032), and the Lianyungang Scientific Plan-Joint Project of Industry-Academia-Research (CXY1405).

\section{References}

[1] S. Kitagawa, R. Kitaura, and S.-I. Noro, "Functional porous coordination polymers," Angewandte Chemie-International Edition, vol. 43, no. 18, pp. 2334-2375, 2004.

[2] J. G. Bünzli and C. Piguet, "Taking advantage of luminescent lanthanide ions," Chemical Society Reviews, vol. 34, no. 12, pp. 1048-1077, 2005.

[3] S. Ma, D. Sun, M. Ambrogio, J. A. Fillinger, S. Parkin, and H.C. Zhou, "Framework-catenation isomerism in metal-organic frameworks and its impact on hydrogen uptake," Journal of the American Chemical Society, vol. 129, no. 7, pp. 1858-1859, 2007.

[4] G. Férey, "Hybrid porous solids: past, present, future," Chemical Society Reviews, vol. 37, no. 1, pp. 191-214, 2008.

[5] J. A. Real, E. Andrés, M. C. Muñoz et al., "Spin crossover in a catenane supramolecular system," Science, vol. 268, no. 5208, pp. 265-267, 1995.

[6] J. W. Han and C. L. Hill, "A coordination network that catalyzes $\mathrm{O}_{2}$-based oxidations," Journal of the American Chemical Society, vol. 129, no. 49, pp. 15094-15095, 2007.

[7] H. A. Habib, J. Sanchiz, and C. Janiak, "Magnetic and luminescence properties of $\mathrm{Cu}(\mathrm{II}), \mathrm{Cu}(\mathrm{II})_{4} \mathrm{O}_{4}$ core, and $\mathrm{Cd}(\mathrm{II})$ mixed-ligand metal-organic frameworks constructed from 1,2bis(1,2,4-triazol-4-yl)ethane and benzene-1,3,5-tricarboxylate," Inorganica Chimica Acta, vol. 362, no. 7, pp. 2452-2460, 2009.

[8] M. Müller, S. Hermes, K. Kähler, M. W. van den Berg, M. Muhler, and R. A. Fischer, "Loading of MOF-5 with $\mathrm{Cu}$ and $\mathrm{ZnO}$ nanoparticles by gas-phase infiltration with organometallic precursors: properties of $\mathrm{Cu} / \mathrm{ZnO} @ \mathrm{MOF}-5$ as catalyst for methanol synthesis," Chemistry of Materials, vol. 20, no. 14, pp. 4576-4587, 2008.

[9] O. R. Evans and W. Lin, "Crystal engineering of NLO materials based on metal-organic coordination networks," Accounts of Chemical Research, vol. 35, no. 7, pp. 511-522, 2002.

[10] J. G. Vitillo, "Magnesium-based systems for carbon dioxide capture, storage and recycling: from leaves to synthetic nanostructured materials," RSC Advances, vol. 5, no. 46, pp. 3619236239, 2015.

[11] N. L. Rosi, J. Eckert, M. Eddaoudi et al., "Hydrogen storage in microporous metal-organic frameworks," Science, vol. 300, no. 5622, pp. 1127-1129, 2003.

[12] M. Eddaoudi, J. Kim, D. Vodak et al., "Geometric requirements and examples of important structures in the assembly of square building blocks," Proceedings of the National Academy of Sciences of the United States of America, vol. 99, no. 8, pp. 49004904, 2002.
[13] M. P. Suh, Y. E. Cheon, and E. Y. Lee, "Syntheses and functions of porous metallosupramolecular networks," Coordination Chemistry Reviews, vol. 252, no. 8-9, pp. 1007-1026, 2008.

[14] B. Chen, C. Liang, J. Yang et al., "A microporous metal-organic framework for gas-chromatographic separation of alkanes," Angewandte Chemie-International Edition, vol. 45, no. 9, pp. 1390-1393, 2006.

[15] J. D. Evans, C. J. Sumby, and C. J. Doonan, "Post-synthetic metalation of metal-organic frameworks," Chemical Society Reviews, vol. 43, no. 16, pp. 5933-5951, 2014.

[16] N. Stock and S. Biswas, "Synthesis of metal-organic frameworks (MOFs): routes to various MOF topologies, morphologies, and composites," Chemical Reviews, vol. 112, no. 2, pp. 933-969, 2012.

[17] A. Pichon, A. Lazuen-Garay, and S. L. James, "Solvent-free synthesis of a microporous metal-organic framework," CrystEngComm, vol. 8, no. 3, pp. 211-214, 2006.

[18] J.-B. Lin, R.-B. Lin, X.-N. Cheng, J.-P. Zhang, and X.-M. Chen, "Solvent/additive-free synthesis of porous/zeolitic metal azolate frameworks from metal oxide/hydroxide," Chemical Communications, vol. 47, no. 32, pp. 9185-9187, 2011.

[19] C. M. Mottillo, Y. Lu, M. H. Pham, M. J. Cliffe, T. Do, and T. Friščić, "Mineral neogenesis as an inspiration for mild, solventfree synthesis of bulk microporous metal-organic frameworks from metal (Zn, Co) oxides," Green Chemistry, vol. 15, no. 8, pp. 2121-2131, 2013.

[20] Z. Ni and R. I. Masel, "Rapid production of metal-organic frameworks via microwave-assisted solvothermal synthesis," Journal of the American Chemical Society, vol. 128, no. 38, pp. 12394-12395, 2006.

[21] O. K. Farha and J. T. Hupp, "Rational design, synthesis, purification, and activation of metal-organic framework materials," Accounts of Chemical Research, vol. 43, no. 8, pp. 1166-1175, 2010.

[22] T. Friščić, I. Halasz, P. J. Beldon et al., "Real-time and in situ monitoring of mechanochemical milling reactions," Nature Chemistry, vol. 5, pp. 66-73, 2013.

[23] J. Klinowski, F. A. Almeida Paz, P. Silva, and J. Rocha, "Microwave-assisted synthesis of metal-organic frameworks," Dalton Transactions, vol. 40, no. 2, pp. 321-330, 2011.

[24] S. T. Meek, J. A. Greathouse, and M. D. Allendorf, "Metalorganic frameworks: a rapidly growing class of versatile nanoporous materials," Advanced Materials, vol. 23, no. 2, pp. 249-267, 2011.

[25] E. R. Parnham and R. E. Morris, "Ionothermal synthesis of zeolites, metal-organic frameworks, and inorganic-organic hybrids," Accounts of Chemical Research, vol. 40, no. 10, pp. 1005-1013, 2007.

[26] G. Lu and J. T. Nupp, "Metal-organic frameworks as sensors: a ZIF-8 based Fabry-Pérot device as a selective sensor for chemical vapors and gases," Journal of the American Chemical Society, vol. 132, no. 23, pp. 7832-7833, 2010.

[27] K. S. Park, Z. Ni, A. P. Cote et al., "Exceptional chemical and thermal stability of zeolitic imidazolate frameworks," Proceedings of the National Academy of Sciences of the United States of America, vol. 103, no. 27, pp. 10186-10191, 2006.

[28] S. Tanaka, K. Kida, T. Nagaoka, T. Ota, and Y. Miyake, "Mechanochemical dry conversion of zinc oxide to zeolitic imidazolate framework," Chemical Communications, vol. 49, no. 72, pp. 7884-7886, 2013.

[29] M. Lanchas, D. Vallejo-Sánchez, G. Beobide et al., "A direct reaction approach for the synthesis of zeolitic imidazolate frameworks: template and temperature mediated control on 
network topology and crystal size," Chemical Communications, vol. 48, no. 79, pp. 9930-9932, 2012.

[30] Y. Pan, Y. Liu, G. Zeng, L. Zhao, and Z. Lai, "Rapid synthesis of zeolitic imidazolate framework-8 (ZIF-8) nanocrystals in an aqueous system," Chemical Communications, vol. 47, no. 7, pp. 2071-2073, 2011.

[31] A. F. Gross, E. Sherman, and J. J. Vajo, "Aqueous room temperature synthesis of cobalt and zinc sodalite zeolitic imidizolate frameworks," Dalton Transactions, vol. 41, no. 18, pp. 54585460, 2012.

[32] A. Lew, P. O. Krutzik, M. E. Hart, and A. R. Chamberlin, "Increasing rates of reaction: microwave-assisted organic synthesis for combinatorial chemistry," Journal of Combinatorial Chemistry, vol. 4, no. 2, pp. 95-105, 2002.

[33] J. A. Gerbec, D. Magana, A. Washington, and G. F. Strouse, "Microwave-enhanced reaction rates for nanoparticle synthesis," Journal of the American Chemical Society, vol. 127, no. 45, pp. 15791-15800, 2005.

[34] K. J. Rao, B. Vaidhyanathan, M. Ganguli, and P. A. Ramakrishnan, "Synthesis of inorganic solids using microwaves," Chemistry of Materials, vol. 11, no. 4, pp. 882-895, 1999.

[35] X. Li, X. Gao, L. Ai, and J. Jiang, "Mechanistic insight into the interaction and adsorption of $\mathrm{Cr}(\mathrm{VI})$ with zeolitic imidazolate framework-67 microcrystals from aqueous solution," Chemical Engineering Journal, vol. 274, pp. 238-246, 2015. 

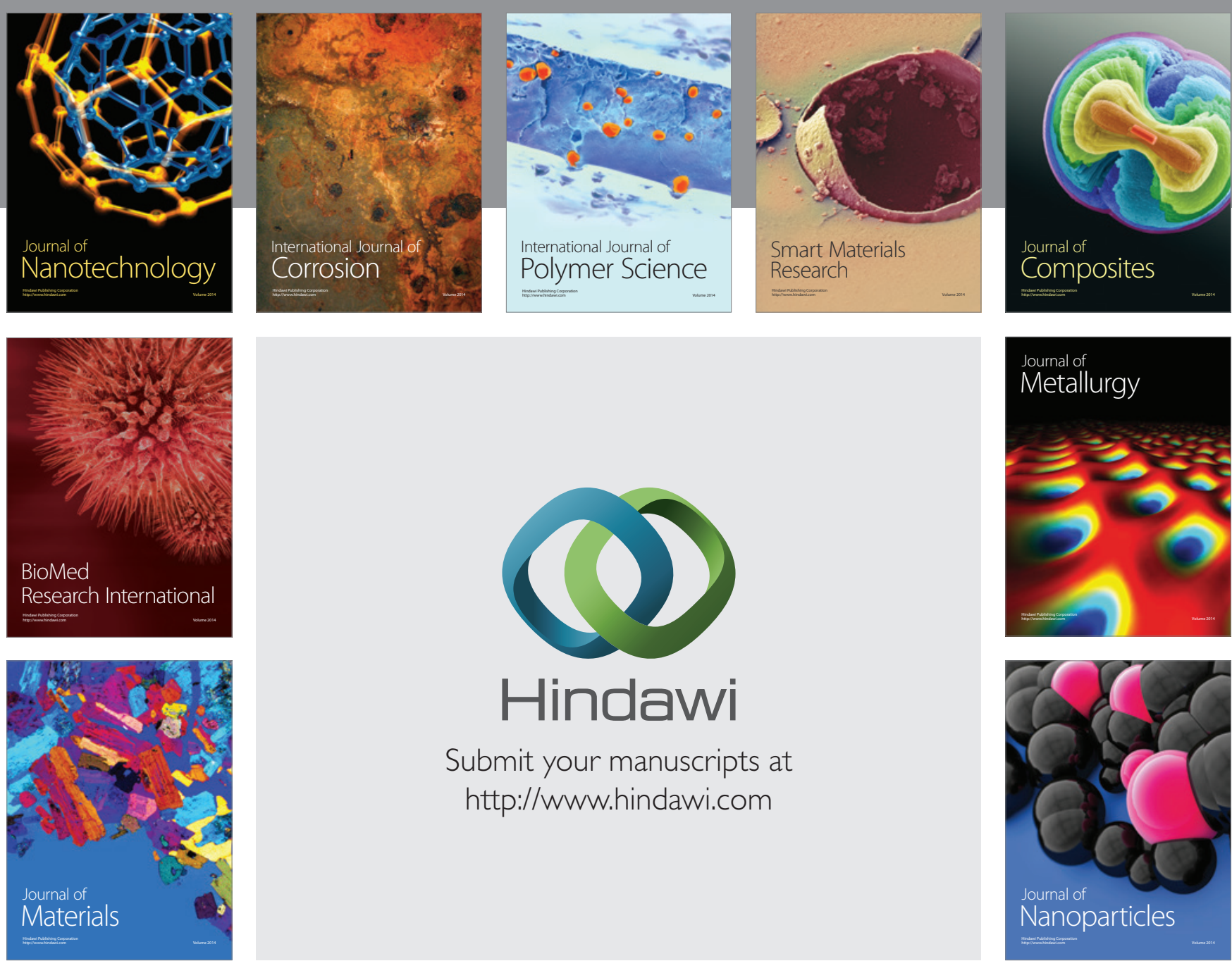

\section{Hindawi}

Submit your manuscripts at

http://www.hindawi.com

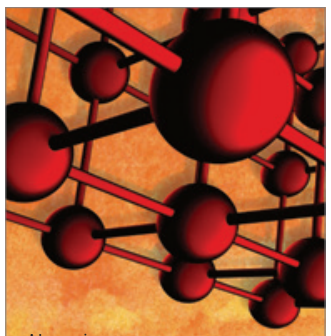

Materials Science and Engineering
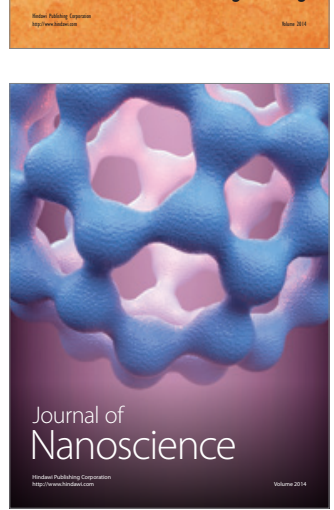
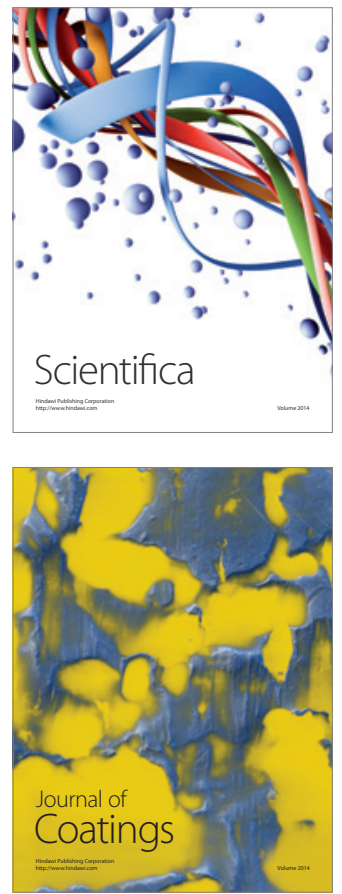
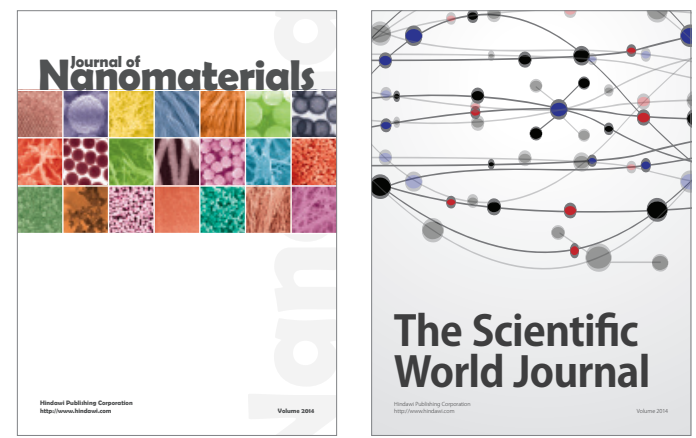

The Scientific World Journal
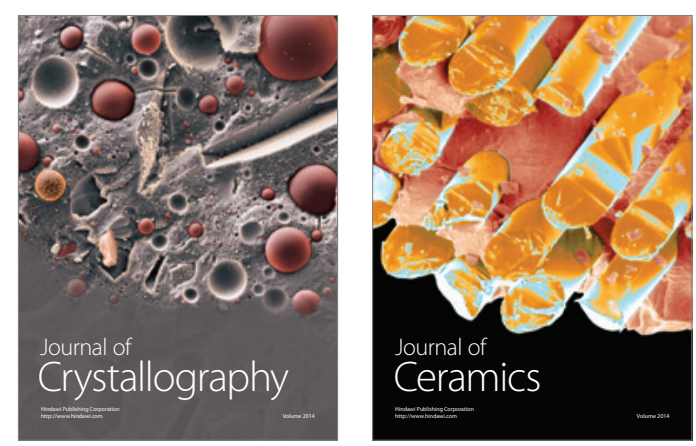
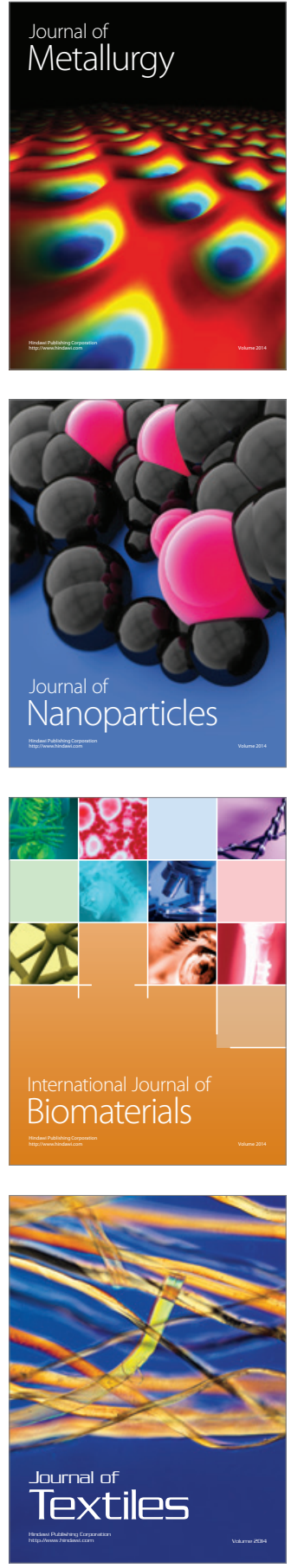